\title{
1 Stress priming affects fungal competition - evidence from a 2 combined experimental and modeling study
}

4 Felix Wesener ${ }^{a}$, Aleksandra Szymczak ${ }^{b}$, Matthias C. Rillig ${ }^{b, c}$, Britta Tietjen ${ }^{a, c}$

6 a Freie Universität Berlin, Institute of Biology, Theoretical Ecology, Königin-Luise-Str. 2/4, Gartehaus, 14195 Berlin,

7 Germany

$8{ }^{b}$ Freie Universität Berlin, Institute of Biology, Ecology of Plants, Altensteinstraße 6, 14195 Berlin, Germany

$9{ }^{c}$ Berlin Brandenburg Institute of Advanced Biodiversity Research (BBIB), 14195 Berlin, Germany 


\section{Abstract}

11 Priming, an inducible stress defense strategy that prepares an organism for an impending stress

12 event, is common in microbes and has been studied mostly in isolated organisms or populations.

13 How the benefits of priming change in the microbial community context and, vice versa, whether

14 priming influences competition between organisms, remains largely unknown. In this combined

15 experimental and modeling study, we developed a cellular automaton model based on dedicated

16 data of different isolates of soil fungi in isolation and pairwise competition experiments. With the

17 model, we simulated growth of the ascomycete Chaetomium elatum competing against other fungi

18 to understand which species traits influence the benefit of priming and the effect of priming on

19 competition. We showed that competition changes the priming benefit compared to isolated

20 growth, and that it depends not only on the primeable species itself, but also on the competitors'

21 traits such as growth rate, primeability and stress susceptibility. In addition, we showed that priming

22 benefits were not always reflected in the competitive outcome. With this study, we transferred

23 insights on priming from studies in isolation to the community context. This is an important step

24 towards understanding the role of inducible defenses in microbial community assembly and

25 composition. 


\section{Introduction}

28 Priming is a stress defense mechanism that enables an organism to remember an environmental

cue and to build up an enhanced stress response to a potentially stronger future stress. Primed

defense mechanisms have been observed across many microbial taxa (see meta-analysis by

Andrade-Linares, Lehmann and Rillig 2016), most of which have focused on the molecular

processes that underlie priming. Complementary to research on priming processes,

understanding the role of priming in stress ecology is an important step to comprehend how

priming might change the effect of stressors on species fitness and community development. At

the ecological level, it is still unclear how the ability of an individual to be primed, termed

primeability, might influence community development and, vice versa, how the community context

37 affects the benefits of priming.

Microbial priming is a defense strategy found in bacteria (Koutsoumanis and Sofos, 2004;

Mitchell et al., 2009; Cebrián et al., 2010; Hernández et al., 2012), archaea (Trent, 1996), as well

as fungi (Alvarez-Peral et al., 2002; Berry and Gasch, 2008; Rangel et al., 2008; Mitchell et al.,

2009; Guhr et al., 2017). Especially fungi are suitable model organisms to study the effects of

priming under different conditions, as many isolates exhibit varying degrees of primeability

(Szymczak et al., 2019) and memory length (Diana R. Andrade-Linares et al., 2016). In nature,

isolated growth of fungi is rare, usually occurring only when new territory is colonized (Boddy,

2000), and fungi normally live in highly complex communities of different species that compete

46 for space and display a broad range of mostly antagonistic interactions (Boddy, 2000; Toljander

47 et al., 2006; Hiscox and Boddy, 2017), which influence community composition (Boddy, 2000, 
species that interact, but also on environmental factors such as resource availability (Stahl and

Christensen, 1992; Falconer et al., 2008) or temperature (Boddy et al., 1985; Schoeman et al.,

1996; Toljander et al., 2006; Hiscox, Clarkson, et al., 2016), and temperature changes can even

lead to reversed competitive outcomes (Crowther et al., 2012). Therefore, we expect that heat

priming, which affects species of distinct primeability differently in their response to heat stress,

has an impact on fungal community development.

Experimental research on priming usually requires time-intensive multifactorial setups, in which

organisms experience, apart from control conditions, a stress with and without preceding priming

cue, as well as a priming cue without subsequent stress (Hilker et al., 2016). Here, simulation

models can complement laboratory experiments by testing different environmental factors and

species traits, e.g. imitating conditions or species combinations that could not be investigated

empirically. A modeling approach thus allows a systematic investigation of distinct costs and

benefits of priming for an organism. Using a mathematical model of microbes in competition, Rillig

et al. (2015) could show that priming is beneficial more often under community conditions

compared to species investigated under isolation. A follow-up study (Wesener and Tietjen, 2019),

additionally showed that different strategies to reach an enhanced stress response are of different

benefit. Especially the stress duration determined if an early or fast buildup of the response was

most beneficial or a stronger response. However, a general understanding of how the benefit of priming can change under competition and how priming influences community structure, is still automaton model simulating the growth of fungal colonies in isolation and in pairwise interactions.

Our model is based on experimental data of the ascomycete Chaetomium elatum as focal species 
stress. It can successfully reproduce the growth dynamics of two competing soil fungi under

primed or non-primed conditions. To gather a general understanding of priming impacts on fungal

communities, we systematically varied different traits of the species competing with C. elatum and

observed how the priming benefit of $C$. elatum changed depending on its competitor. The specific

aims of the study are i) to identify fungal traits that affect the pay-off of priming by comparing the

benefit of $C$. elatum in dual cultures with various competitors and ii) to assess the influence of

priming on competitive success.

\section{Methods}

We carried out an experiment on six soil fungal species growing in isolation and in dual-species

settings to determine the intrinsic growth rate of each species measured as colony diameter

extension. For these settings, we determined how the growth of species is altered by heat stress

and by priming towards this stress. We used parts of the data (growth under different stress

treatments in isolation and unstressed growth under competition) to parameterize growth rates of

the model. We then validated our model by comparing the model output with the experimental data

on dual-culture growth under stress and priming. Finally, we used the validated model to

systematically assess the effect of fungal species traits and of competition on the benefits of

priming. Moreover, the model served to investigate how priming influences competition between

89 species.

Experimental setup

91 For the laboratory experiment, six soil fungal species (Ascomycetes Chaetomium elatum, Fusarium

92 redolens, Fusarium oxysporum and Truncatella angustata, Basidiomycete Pleurotus sapidus and

93 Mucoromycete Morteriella elongata) were grown in isolation and in competition in a full-factorial 
design of priming and triggering for heat stress. The soil saprotrophic fungi were originally taken

from a grassland site in Mallnow (Mallnow Lebus, Brandenburg, Germany, 52²7.778' N, 14²9.349'

non-detrimental heat stress of $35^{\circ} \mathrm{C}$ for two hours, as triggering stimulus, we used a temperature of

treatments: i) A control treatment (C), simulating constant conditions of $22{ }^{\circ} \mathrm{C}$ with no disturbance,

ii) a priming-only treatment $(P)$, in which a fungus experienced the priming stimulus after one day of

followed by a triggering stimulus.

For the single-species experiments, the colony diameter was measured once per day to determine edges of the Petri dish or, for slower growing individuals, for 14 consecutive days. pairwise with each of the five other species equally distant from each other and from the border of

112 the Petri dish. As soon as the two colonies touched, the same treatments on priming and triggering

113 as in the single species experiments were applied. In pairwise cultures, the individual colony shapes were not circular. Therefore, instead of diameter measurements the surface area of each colony was 
117 Eight replicates per fungus/competition setup and treatment were measured.

119 We determined the species-specific growth rates by measuring the change of colony diameter

120 (single species) or colony area (dual cultures) over time in all four experimental treatments. We

121 compared the growth in the $\mathrm{C}$ and $\mathrm{P}$ treatment to determine whether a priming stimulus in itself

122 would already reduce fungal growth. A significant reduction of growth in the P treatment would

123 imply that priming inflicts costs on a primed species on the level of growth, in case a priming

124 stimulus was not followed by a triggering stress. The comparison between $\mathrm{C}$ and T treatment

125 revealed the effect of stress on growth, while comparing PT and T treatment quantified how much

126 better a species grows, if primed before stressed.

127 For the $\mathrm{C}$ and $\mathrm{P}$ treatment, a linear fit was applied to the daily diameter values of the

128 single species experiments resulting in colony growth measured as colony diameter

129 change [mm/day]. For the T and PT treatments, we detected the occurrence of a stress-

130 induced lag phase, i.e. a period of no growth, and determined the growth of the

131 following phase. To do so, we compared the colony diameter distribution of the single

132 species replicates on day 1 (day of treatment) with each respective following day using

133 a paired t-test after testing for normality and homoscedasticity. We measured the

134 duration of the lag phase $L_{S i, T}$ and $L_{S i, P T}$ as the time period following a T and PT

135 treatment, in which the colony diameter does not differ significantly from day 1.

136 Growth rates were determined for the post-lag growth phase with a linear fit. The exact

137 time point of transition from lag phase to growth was the intersection of the lag and

138 growth fit lines. 
139 Of the six species that were primed experimentally, four did not show a significant

140 difference in growth between control and priming treatment, i.e. no priming costs occurred

141 on the level of growth (Table 1). The other two species, F. oxysporum and T. angustata,

142 showed a slight overall increase in growth. To reduce the complexity of our model, we thus

143 chose to exclude priming costs for further analyses. The effects of heat stress were

144 similar across all species but two: all fungi exhibited a lag phase without any growth, and

145 four of the six species did not show a change in growth rate after the lag had ended

146 compared to unstressed growth. Solely T. angustata showed growth that was different by

147 more than $10 \%$ and even higher than under control conditions, while F. oxysporum showed

148 a slight reduction in growth after the lag phase.

149 When being primed, the lag phase after experiencing stress was shorter in five species or

150 remained equal in $M$. elongata, and the growth rate after the end of the lag phase did not

151 differ. Again, to avoid unnecessary model complexity, we assumed no difference in the

152 growth rate after stress-induced lag phases for both T and PT treatments.

Table 1

\begin{tabular}{|c|c|c|c|c|c|c|}
\hline Species & $\begin{array}{c}\text { Control } \\
\text { growth rate } \\
g_{s i}[\mathrm{~mm} / \mathrm{d}]\end{array}$ & $\begin{array}{c}\text { Rel. change } \\
\text { in growth } \\
T / C\end{array}$ & $\begin{array}{c}\text { Rel. change } \\
\text { in growth } \\
\mathrm{PT} / \mathrm{T}\end{array}$ & $\begin{array}{c}\text { Rel. change } \\
\text { in growth } \\
P / C\end{array}$ & $\begin{array}{c}\text { Non-primed } \\
\text { lag phase } L_{S i, T} \\
\text { [d] }\end{array}$ & $\begin{array}{c}\text { Primed lag } \\
\text { phase } \\
L_{S i, P T}[\mathrm{~d}]\end{array}$ \\
\hline C. elatum & 9.38 & 1 & 1 & 1 & 3.17 & 1.9 \\
\hline M. elongata & 14.74 & 1 & 1 & 1 & 2.82 & 2.82 \\
\hline F. oxysporum & 10.57 & $0.91(* *)$ & 1 & $1.046(*)$ & 0.55 & 0.12 \\
\hline F. redolens & 6.63 & 1 & 1 & 1 & 0.71 & 0.01 \\
\hline P. sapidus & 3.58 & 1 & 1 & 1 & 1.93 & 1.56 \\
\hline T. angustata & 7.13 & $1.15(* *)$ & 1 & $1.10(*)$ & 1.577 & 1.03 \\
\hline
\end{tabular}


158 To simulate a fungal colony growing in a Petri dish, we developed a cellular automaton model and introduced the experimentally determined growth rates into this model. In

160 the following section, we describe the model and how we converted the measured growth

161 rates of the experiment, which are constant over time and space, to the necessary

162 discrete units of time and space of the cellular automaton.

163 Our model represents a Petri dish, i.e. a circular area, with an inner diameter $d=86.5$

$164 \mathrm{~mm}$ containing one or two fungal colonies. The area of the Petri dish is divided into

165 square grid cells with a side length of $r_{\text {spat }}=0.5 \mathrm{~mm}$, leading to 173 grid cells along

166 the diameter of the Petri dish. To mimic the laboratory experiments, the initial colony

167 diameter of a fungus is set to $d=6 \mathrm{~mm}$. Colonies in isolation are placed into the center

168 of the Petri dish. Colonies in pairwise experiments are placed on the horizontal

169 diameter equidistant from each other and the border of the Petri dish, i.e. the distance

170 between the centers of the colonies is about $28.5 \mathrm{~mm}$.

171 Simulation of cell division and colony growth follows the cellular automaton model of

172 Gerlee and Anderson (2007). To realize radial extension of the initial colonies, each grid

173 cell of the model is assigned one of three states: empty, occupied by an active (growing)

174 fungal cell or occupied by a dormant fungal cell. Fungal cells with empty neighboring grid

175 cells (neighbors being all grid cells in the Moore neighborhood of a respective cell) that

176 could thus still spread are considered active cells, while fungal cells without any empty

177 neighboring grid cells turn dormant. Only active fungal cells conduct cell division, i.e. 
178 produced new daughter cells on one of the empty neighboring grid cells, leading to an

179 increase in colony area. Therefore, active fungal cells can solely be found in the

180 periphery of a fungal colony. Each time step, the active fungal cells are updated and

181 behaved accordingly, i.e. active cells that are no longer in the colony circumference

182 change to the dormant state.

183 The temporal resolution $r_{\text {temp }}$ is one hour. To match measured growth rates, it is

184 necessary to determine the frequency of cell division, for which we introduce a linear

185 increasing maturation value $m(t)$ for each active cell. Cell division occurs when a cell

186 reaches a maturation age of $m \geq 1$. The increase in $m, \Delta m$, is calculated based on the

187 measured growth rate $g_{s i}$ relative to the temporal and spatial resolution:

$$
\Delta m=\left\{\begin{array}{cc}
0, & t_{\text {treatment }}<t<t+L_{\text {si,treatment }} \\
\frac{g_{\text {si }} \cdot r_{\text {temp }}}{2 \cdot r_{\text {spat }}}, & \text { else }
\end{array}\right.
$$

with si referring to the simulated species and treatment referring to the primed only or primed and 
an increase in area. The maturation value is then reduced by 1 . The average division number corresponds to the measured radial colony extension. The daughter cell inherits its mother's new maturation age adjusted by a random variation term with a standard deviation of $\sigma=m / 2$. If at the point of division none of the neighboring grid cells is empty, the division failed and the fungal cell changes to the dormant state.

Apart from competition for space, no other forms of interactions are included. The model

space between competing species and the most common competitive outcome of mycelial interactions (Stahl and Christensen, 1992; Schoeman et al., 1996; Hiscox et al., 2018).

The cellular automaton model was implemented in NetLogo 6.1.0 (Wilensky, 1999) and analysed using R (R., 2018) and the nlrx package (Salecker et al., 2019).

\section{Model Parameterization}

212 The following model parameters are based on experimental data of single-species and are specific for each species si: i) The intrinsic colony extension rate in isolation $g_{s i}[\mathrm{~mm} / \mathrm{day}]$, ii) the stress susceptibility defined as the length of the fungistatic lag phase under heat stress $s u s_{s i}=L_{s i, T}$, and iii) the primeability of a species describing the reduction of the lag phase if primed before

216 stressed $\operatorname{prim}_{s i}=1-\frac{L_{s i, P T}}{L_{s i, T}}$. A primeability value of $\operatorname{prim}_{s i}=1$ describes full primeability, i.e. a

217 reduction of the lag phase to zero, while a primeability of prim $_{s i}=0$ applies to non-primeable 218 species that exhibit the same lag phase under T and PT treatment.

219 Because fungi change their growth rates in dual cultures depending on their competitor (Stahl

220 and Christensen 1992), we adjusted the growth rate $g_{s i, c i}$ of species si under competition with

221 competitor ci by re-calibrating growth to match the growth observed in the dual culture 
control experiments. To determine the change in growth rates under competition, we applied a

223 fit to non-stressed conditions, and only on data points before both fungal colonies touched.

224 With this, we can differentiate the effect that arises due to interactions at distance from the

225 effect of direct competition for space. The latter is already implemented in the model, as

226 simulated fungal cells can only colonize empty grid cells. All parameters are given in

227 Table 2.

228

229

Table 2

\begin{tabular}{|c|c|c|c|}
\hline Parameter & Description & Source & Unit \\
\hline$g_{s i}$ & intrinsic colony extension rate in isolation & measured & $\underline{\mathrm{mm} / \mathrm{d}}$ \\
\hline$g_{s i, c i}$ & intrinsic colony extension rate under competition & measured & $\mathrm{mm} / \mathrm{d}$ \\
\hline$L_{S i, T}$ & duration of a phase of no growth after a $2 \mathrm{~h}$ heat stress & measured & $d$ \\
\hline$L_{S i, P T}$ & $\begin{array}{l}\text { duration of a lag phase of no growth if primed before } \\
\text { stressed }\end{array}$ & measured & $d$ \\
\hline $\operatorname{sus}_{s i}$ & stress susceptibility & $s u s_{s i}=L_{s i, T}$ & $d$ \\
\hline prim $_{s i}$ & $\begin{array}{l}\text { stress primeability (reduction of the stress-induced lag } \\
\text { phase if primed) }\end{array}$ & $\operatorname{prim}_{s i}=1-\frac{L_{s i, P T}}{L_{s i, T}}$ & - \\
\hline
\end{tabular}

230

232 To validate the model, we simulated the growth of $C$. elatum as focal species in isolation and

233 under competition with each one of the other five fungi under stress treatment $(T)$ and primed-

234 and-stressed treatment (PT). As model output, we determined the colony area of $C$. elatum

$235 A_{\text {C.elatum }}=N_{\text {cells }} \cdot r_{\text {spat }} 2\left[\mathrm{~mm}^{2}\right]$, which serves as a relative measure of fitness. The benefit of

236 priming for C. elatum was then described as the colony area of a fungus under stress (T) compared

237 to the area of a primed colony under stress (PT): 


$$
b_{s i}=\frac{A_{s i, P T}}{A_{s i, T}}
$$

To assess model performance, the colony growth of $C$. elatum with each of its respective

240 competitors as well as the simulated benefit was compared to experimental data.

241 Subsequently, we performed simulation experiments to determine which species-specific traits

242 influence the benefit of priming and the competition between two species under priming. First, we

243 simulated the growth of $C$. elatum in competition with an artificial species initially exhibiting the

244 same trait values as C. elatum until the Petri dish was filled or up to a maximum of 15 days. To

245 examine the effect of specific traits and trait combinations on the priming benefit, we

systematically varied the growth rate, stress susceptibility and primeability values of the competitor

247 and measured the benefit $b$ of $C$. elatum. Because the growth of $C$. elatum under competition

248 proved to be variable depending on its competitor, we also varied the growth rate of $C$. elatum,

249 while all other trait values of $C$. elatum remained fixed.

250 Secondly, to determine the effect of priming on a simple community, i.e. which competitor benefits

251 more from priming, we measured the relative benefit

$$
b_{\text {rel,C.elatum }}=\frac{A_{\text {C.elatumPT }}}{A_{\text {C.elatumT }}} / \frac{A_{\text {competitorPT }}}{A_{\text {competitorT }}}
$$

, which compares the benefit of both competitors.

$$
c_{\text {C.elatum }}=\ln \left(\frac{A_{\text {C.elatumPT }}}{A_{\text {competitorPT }}}\right)-\ln \left(\frac{A_{\text {C.elatumT }}}{A_{\text {competitorT }}}\right)
$$


260 which describes the influence of priming on competition for space: Having experienced a priming

261 cue before the triggering stress, one species might benefit more than the other leading to a shift in

262 the colony ratio compared to the same scenario without priming cue. A value of $c_{s i}=0$ refers to

263 no change in colony ratios, i.e. both competitors benefit equally from priming. For a value of $c_{s i}>$

2640 , the colony size of $C$. elatum increases more than the one of its competitor. Measuring both, the

265 benefit of $C$. elatum and the competitive shift $c_{\text {C.elatum, allowed us to investigate whether certain }}$

266 parameter combinations affected these values differently, e.g. led to high priming benefit of $C$.

267 elatum but still decreased its competitive strength because the competitor benefitted even more.

\section{Results}

269 After model fitting, we first validated the model by comparing the simulated output with

270 experimental data of competition treatments not used for model parameterization. We then

271 systematically varied different traits of an artificial species competing with C. elatum and assessed

272 the benefit that C. elatum gained from priming. Additionally, we measured the effect of priming

273 on competition strength under stress conditions.

275 With our model, we could well predict the growth in competition of four of five fungal pairs under

276 stress with and without preceding priming cue (see Fig. 1 and Fig. S1). In these successful cases, the

277 effects of interactions and stress were additive. When competing with $M$. elongata, however, the

278 model underestimated the performance of C. elatum: While under control conditions, M. elongata

279 overgrew C. elatum in the experiment and thus dominated strongly, under stress, M. elongata

280 changed its behavior and could no longer overgrow $C$. elatum. This led to a stronger benefit of $C$. 
elatum instead of the expected additive effects. For the sake of simplicity, however, our model does not yet take into account interactions between competition and stress nor alternative forms of fungal interactions such as overgrowth.

The priming benefit of $C$. elatum predicted by the simulation model was within the range of variation of the observed benefit for all five pairs (Fig. 2b). By comparing T and PT treatment, the benefit quantifies the effect of priming, canceling out stress effects: The underestimation of the performance of $C$. elatum competing with $M$. elongata thus does not affect the model prediction on priming benefits.

290 When simulating the development of priming benefits over time, a consistent pattern emerged for both isolated and competitive growth (Fig. 2a): The benefit increased just after primed C. elatum restarted growth after the lag phase, and reaches a maximum when the non-primed lag phase ended. The subsequent decrease in benefit results from the simultaneous increase each colony's areas leading to a smaller relative difference between their area.

For isolated growth, the immediate benefit was larger than under competition, because an isolated colony could expand unimpeded and benefit strongly from the shortened lag phase, while under competition, this space might be already occupied by a competitor. A competitor would have

298 already claimed part of the space a species could grow on. The final benefit, however, was lowest

299 in isolation, because without competitors there was no advantage in claiming space earlier, as

300 eventually all available space would be overgrown. This final benefit was largest when C. elatum

301 faced one of the two most competitive, i.e. fastest growing, species investigated: M. elongata, 302 which exhibits no primeability, followed by F. oxysporum. 
When we systematically varied fungal traits, for all combinations of traits within the investigated

parameter space, priming was beneficial (i.e. benefit >1, Fig. 3) eight days after inoculation, since

priming involved no costs. However, under competition with a highly primeable and stress-

susceptible competitor, priming was only marginally beneficial, especially when the competing species

was fast-growing. Conversely, we observed the highest benefit when C. elatum faced a stress-

resistant and only moderately primeable competitor. Here, the negative effect of fast growing

competitors was reversed (upper right vs. lower left panel of Fig. 3). A very susceptible competitor

with high primeability strongly reduced its lag phase under priming. The faster that opponent

grows, the more C. elatum will suffer from its gain in growing time. If, however, the opponent is

not very primeable, a priming cue will be of no great advantage to that species. In this case, $C$.

elatum will benefit even more if the competitor is fast-growing.

Fifteen days after inoculation, when the Petri dish was filled and a steady state was reached,

priming was not beneficial (i.e. benefit $<1$ ) in case of a fast growing, highly primeable competitor

with intermediate or high stress susceptibility (Fig. S2). During phases of growth, space that is lost

to a more primeable competitor can still be compensated by colonizing empty space elsewhere. In

this case, it can be more beneficial not to be primed at all, if space is limited.

320 Analogous to the investigation of priming benefits, we measured how the colony ratio between $C$.

323 favorable conditions arose when C. elatum faced stress-susceptible but primeable opponents.

324 However, because of its intermediate primeability, for most investigated trait combinations, $C$. 
elatum benefited stronger than its competitor, as the long stress-induced lag phase of $C$. elatum is

326 reduced substantially.

327 When comparing figure 3 and 4 , it is notable that while in many cases priming conferred a

328 moderate benefit to $C$. elatum, the competitive shift in these cases was variable: facing a

329 primeable but stress susceptible and slow competitor, C. elatum moderately benefitted from

330 priming. However, since the competitor's benefit is higher, the colony ratio was shifted in favor of

331 the competitor. If on the other hand the competitor was less primeable, the absolute benefit of $C$.

332 elatum was similar as before, but this time leading to a positive shift in the colony ratios in favor of

333 C. elatum.

\section{Discussion}

335 We successfully developed a cellular automaton model that reproduces growth of competing fungi

336 in a Petri dish under priming and heat stress conditions. With this model, we assessed how different

337 fungal traits such as stress susceptibility and primeability influence the species-specific benefit and

338 competition outcomes.

339 The priming response of fungi

340 For all experimentally treated species, a heat-induced no-growth phase was observed in the

341 experimental data, and for four of six species, the post-lag growth phase was not significantly

342 different from the control growth. Priming did not affect the growth of any of the species, but

343 instead reduced the duration of the phase without growth.

344 An analytical study by Wesener and Tietjen (2019) using coupled differential equations of microbial

345 growth showed that stress of short duration is best met with an early defense and that a primed 
stress strategy is most successful when further shortening the response. The current study confirms this pattern, as the fungi were treated with two-hour pulses of heat instead of prolonged periods of warming, and the primed colonies restarted growth earlier than those that had not been primed.

between an immediate but slow reversal to control level growth or a lag phase with no growth and

the fungal stress response types differ for different types of heat stress.

In all cases, heat stress affected growth. Additionally, in some species combinations it could also

deadlock. This is in line with previous findings (Hiscox, Clarkson, et al., 2016) and could be a

valuable extension to our simulation model.

\section{Priming costs}

In this study, we aimed at accurately imitating growth dynamics of fungi under priming conditions.

We ended at not implementing any costs of priming, as there was little evidence under laboratory

367 The distribution of resources between growth, resistance and reproduction is central to ecological 
369 Crowther et al., 2014). A priming mechanism without costs would not bear any risks, and even in

370 environments with low stress predictability (leading to organisms reacting to a priming cue, which

371 is not followed by a triggering stress), priming would be of no disadvantage and would be ubiquitous

372 in nature. To our knowledge, there is no study that investigated the costs of priming in microbes.

373 Studies on priming costs in plants differed in their results for different species and priming cues,

374 finding no direct costs of priming (Perazzolli et al., 2011), costs realized as growth reduction (Hulten

375 et al., 2006), or reduced rhizome production (Yip et al., 2019). Priming costs in fungi might thus also

376 not be manifested in reduced growth, but rather in reduced spore production or competitive

377 strength. Therefore, we want to stress the need of research on costs of induced resistance in microbes,

378 which is necessary to fully comprehend the benefits and potential trade-offs of priming.

379 The benefit of priming

380 Because we did not implement any priming costs, during the growth phase priming is generally

381 beneficial for $C$. elatum in all investigated scenarios. Therefore, we focus rather on the magnitude

382 and not on the presence of this benefit.

383 Our results show that the benefit of priming under competition is highly dependent on fungal traits such as

384 primeability, stress susceptibility and growth, as well as the time point during community buildup. We could

385 show that depending on these factors, priming might not always more beneficial under competition compared

386 to the isolated benefit. Even when priming itself is of low direct benefit for a given species, it can still

387 shift dominance in favor of that particular species, depending on the traits of its competitors.

388 Priming can influence competition between species by shifting dominance towards one of the

competitors, especially favoring primeable species that otherwise show a competitive disadvantage

390 (i.e. not stress-resistant or slow-growing). Priming can thus be beneficial when taking into account 
the change of the community structure and the resulting fitness of competing species, making it

392 difficult to infer priming effects in a community from effects measured on species in isolation.

C. elatum exhibits moderate primeability and shows the longest stress-induced lag phase of the six

394 investigated species. As a result, priming has the potential to strongly shorten its lag phase and thus

395 to be highly beneficial in comparison to its competitors with lower susceptibility or lower

396 primeability. Because the competition for space in fungal communities is effectively competition for

397 gaining access to nutrients (Boddy, 2000), it is of particular importance when recolonizing new

398 territory. A primed stress response that allows an organism to occupy empty space earlier than its

399 competitors therefore leads to the additional advantage of claiming space that would otherwise be

400 colonized by another species. The order of species arrival in community assembly affects

401 community structure and function (Fukami, 2015) and priority effects have been shown to be a

402 common influence on fungal communities (Kennedy et al., 2009). These priority effects can even

403 be of increased importance when species further change environmental conditions or resource

404 availability for later species via niche modification (Fukami, 2015). Environmental factors such as

405 temperature have been shown to influence assembly of fungal community members (Hiscox et al.,

406 2015; Hiscox, Clarkson, et al., 2016; Hiscox, Savoury, et al., 2016). Therefore, heat priming can

407 potentially influence the order of community assembly by letting certain species grow earlier than

408 others.

409 Priming might not only affect community composition via community assembly, but also directly

410 influence community structure: Sensitivity of microbial communities to disturbances is common, as

411 they rarely return to pre-disturbance composition and reach alternative stable states (Shade et al.

412 2012; Schimel, Balser, and M. Wallenstein 2007; Allison and Martiny 2008). Environments with

413 fluctuating temperature increase species number in fungal communities (Toljander et al., 2006), 
and post-stress communities can transiently consist of species that are generally more resistant to stress because of their higher likelihood to survive past stress events (Evans and Wallenstein, 2012;

416 Jurburg et al., 2017). Priming, however, can influence community resistance, if less resistant but

417 instead primeable species persist in a community. Stress responses at an individual level, such as priming, might therefore interact with legacy effects arising from pre-disturbance community

419 composition (Meisner et al., 2018), resulting in communities with different functions or stress 420 resistance.

421 With this study, we could show that the effect of priming on a species and on the community 422 structure is not consistent, but highly varies in strength depending on the primeability and stress susceptibility of other members in the community. It is therefore essential to understand how the community context and different species traits affect priming to comprehend its influence on community assembly. We therefore want to stimulate further research on inducible stress defenses

426 in microbes and their effects on community development, as well as the effect of the community context on stress defense mechanisms.

429 Acknowledgements

430 We are grateful to the German Research Foundation (DFG) for funding our Collaborative Research 431 Centre 973 'Priming and Memory of Organismic Responses to Stress' (www.sfb973.de). 


\section{References}

Alvarez-Peral, F.J., Zaragoza, O., Pedreno, Y., and Argüelles, J.-C. (2002) Protective role of trehalose during severe oxidative stress caused by hydrogen peroxide and the adaptive oxidative stress response in Candida albicans. Microbiology 148: 2599-2606.

Andrade-Linares, Diana R., Lehmann, A., and Rillig, M.C. (2016) Microbial stress priming: a metaanalysis. Environ Microbiol 18: 1277-1288.

Andrade-Linares, Diana R., Veresoglou, S.D., and Rillig, M.C. (2016) Temperature priming and memory in soil filamentous fungi. Fungal Ecol 21: 10-15.

Berry, D.B. and Gasch, A.P. (2008) Stress-activated Genomic Expression Changes Serve a Preparative Role for Impending Stress in Yeast. Mol Biol Cell 19: 4580-4587.

Boddy, L. (2001) Fungal Community Ecology and Wood Decomposition Processes in Angiosperms : From Standing Tree to Complete Decay of Coarse Woody Debris. Ecol Bull 43-56.

Boddy, L. (2000) Interspecific combative interactions between wood-decaying basidiomycetes. FEMS Microbiol Ecol 31: 185-194.

Boddy, L., Gibbon, O.M., and Grundy, M.A. (1985) Ecology of Daldinia concentrica: effect of abiotic variables on mycelial extension and interspecific interactions. Trans - Br Mycol Soc 85: 201211.

Cebrián, G., Sagarzazu, N., Pagán, R., Condón, S., and Mañas, P. (2010) Development of stress resistance in Staphylococcus aureus after exposure to sublethal environmental conditions. Int J Food Microbiol 140: 26-33.

Crowther, T.W., Littleboy, A., Jones, T.H., and Boddy, L. (2012) Interactive effects of warming and invertebrate grazing on the outcomes of competitive fungal interactions. FEMS Microbiol Ecol 81: 419-426.

Crowther, T.W., Maynard, D.S., Crowther, T.R., Peccia, J., Smith, J.R., and Bradford, M.A. (2014) Untangling the fungal niche: the trait-based approach. Front Microbiol 5: 1-12.

Evans, S.E. and Wallenstein, M.D. (2012) Soil microbial community response to drying and rewetting stress: does historical precipitation regime matter? Biogeochemistry 109: 101-116.

Falconer, R.E., Bown, J.L., White, N.A., and Crawford, J.W. (2008) Modelling interactions in fungi. J R Soc Interface 5: 603-615. 
Fukami, T. (2015) Historical Contingency in Community Assembly: Integrating Niches, Species Pools, and Priority Effects. Annu Rev Ecol Evol Syst 46: 1-23.

Gerlee, P. and Anderson, A.R.A. (2007) Stability analysis of a hybrid cellular automaton model of cell colony growth. Phys Rev E - Stat Nonlinear, Soft Matter Phys 75: 1-8.

Guhr, A., Horn, M.A., and Weig, A.R. (2017) Vitamin B 2 (Riboflavin) increases drought tolerance of Agaricus bisporus. Mycologia 109: 860-873.

Harvell, C.D. (1990) The ecology and evolution of inducible defenses. Q Rev Biol 65: 323-340.

Heil, M. (2002) Ecological costs of induced resistance. Curr Opin Plant Biol 5: 345-350.

Heil, M. (2014) Trade-offs Associated with Induced Resistance. In, Induced Resistance for Plant Defense. Chichester, UK: John Wiley \& Sons, Ltd, pp. 171-192.

Hernández, S.B., Cota, I., Ducret, A., Aussel, L., and Casadesús, J. (2012) Adaptation and Preadaptation of Salmonella enterica to Bile. PLoS Genet 8: e1002459.

Hilker, M., Schwachtje, J., Baier, M., Balazadeh, S., Bäurle, I., Geiselhardt, S., et al. (2016) Priming and memory of stress responses in organisms lacking a nervous system. Biol Rev 91: 11181133.

Hiscox, J. and Boddy, L. (2017) Armed and dangerous - Chemical warfare in wood decay communities. Fungal Biol Rev 31: 169-184.

Hiscox, J., Clarkson, G., Savoury, M., Powell, G., Savva, I., Lloyd, M., et al. (2016) Effects of precolonisation and temperature on interspecific fungal interactions in wood. Fungal Ecol 21: 3242.

Hiscox, J., O'Leary, J., and Boddy, L. (2018) Fungus wars: basidiomycete battles in wood decay. Stud Mycol 89: 117-124.

Hiscox, J., Savoury, M., Johnston, S.R., Parfitt, D., Müller, C.T., Rogers, H.J., and Boddy, L. (2016) Location, location, location: priority effects in wood decay communities may vary between sites. Environ Microbiol 18: 1954-1969.

Hiscox, J., Savoury, M., Müller, C.T., Lindahl, B.D., Rogers, H.J., and Boddy, L. (2015) Priority effects during fungal community establishment in beech wood. ISME J 9: 2246-2260.

Hulten, M. van, Pelser, M., Loon, L.C. van, Pieterse, C.M.J., and Ton, J. (2006) Costs and benefits of priming for defense in Arabidopsis. Proc Natl Acad Sci 103: 5602-5607.

Jurburg, S.D., Nunes, I., Brejnrod, A., Jacquiod, S., Priemé, A., Sørensen, S.J., et al. (2017) Legacy 

Perturbation. Front Microbiol 8: 1832.

Kennedy, P.G., Peay, K.G., and Bruns, T.D. (2009) Root tip competition among ectomycorrhizal fungi: Are priority effects a rule or an exception? Ecology 90: 2098-2107.

Koutsoumanis, K.P. and Sofos, J.N. (2004) Comparative acid stress response of Listeria monocytogenes, Escherichia coli 0157:H7 and Salmonella Typhimurium after habituation at different pH conditions. Lett Appl Microbiol 38: 321-326.

Meisner, A., Jacquiod, S., Snoek, B.L., Ten Hooven, F.C., and van der Putten, W.H. (2018) Drought legacy effects on the composition of soil fungal and prokaryote communities. Front Microbiol

R. (2018) R core team (2018). R A Lang Environ Stat Comput R Found Stat Comput Vienna, Austria

Rangel, D.E.N., Anderson, A.J., and Roberts, D.W. (2008) Evaluating physical and nutritional stress 9: $1-12$.

Mitchell, A., Romano, G.H., Groisman, B., Yona, A., Dekel, E., Kupiec, M., et al. (2009) Adaptive prediction of environmental changes by microorganisms. Nature 460: 220-224.

Perazzolli, M., Roatti, B., Bozza, E., and Pertot, I. (2011) Trichoderma harzianum T39 induces resistance against downy mildew by priming for defense without costs for grapevine. Biol Control 58: 74-82.

Salecker, J., Sciaini, M., Meyer, K.M., and Wiegand, K. (2019) The nlrx r package: A next-generation framework for reproducible NetLogo model analyses. Methods Ecol Evol 10: 1854-1863.

Schneider, C.A., Rasband, W.S., and Eliceiri, K.W. (2012) NIH Image to ImageJ: 25 years of image analysis. Nat Methods 9: 671-675. during mycelial growth as inducers of tolerance to heat and UV-B radiation in Metarhizium anisopliae conidia. Mycol Res 112: 1362-1372.

Rillig, M.C., Rolff, J., Tietjen, B., Wehner, J., and Andrade-Linares, D.R. (2015) Community priming-effects of sequential stressors on microbial assemblages. FEMS Microbiol Ecol 91: 1-7.

Schoeman, M.W., Webber, J.F., and Dickinson, D.J. (1996) The effect of diffusible metabolites of Trichoderma harzianum on in vitro interactions between basidiomycete isolates at two different temperature regimes. Mycol Res 100: 1454-1458.

Schulenburg, H., Kurtz, J., Moret, Y., and Siva-Jothy, M.T. (2009) Introduction. Ecological 
immunology. Philos Trans R Soc B Biol Sci 364: 3-14.

525 Stahl, P.D. and Christensen, M. (1992) In vitro mycelial interactions among members of a soil 526 microfungal community. Soil Biol Biochem 24: 309-316.

527 Szymczak, A., Ryo, M., and Rillig, M.C. (2019) Diversity of responses of soil saprobic fungi to 528 recurring heat events.

529 Toljander, Y.K., Lindahl, B.D., Holmer, L., and Högberg, N.O.S. (2006) Environmental fluctuations

530 facilitate species co-existence and increase decomposition in communities of wood decay 531 fungi. Oecologia 148: 625-631.

532 Trent, J.D. (1996) A review of acquired thermotolerance, heat-shock proteins, and molecular 533 chaperones in archaea. In, FEMS Microbiology Reviews., pp. 249-258.

534 Wesener, F. and Tietjen, B. (2019) Primed to be strong, primed to be fast: modeling benefits of 535 microbial stress responses. FEMS Microbiol Ecol 95: 1-9.

536 Wilensky, U. (1999) NetLogo. http://ccl.northwestern.edu/netlogo/. Cent Connect Learn Comput Model Northwest Univ Evanst.

538 Yip, E.C., Tooker, J.F., Mescher, M.C., and De Moraes, C.M. (2019) Costs of plant defense priming:

539 Exposure to volatile cues from a specialist herbivore increases short-term growth but reduces $540 \quad$ rhizome production in tall goldenrod (Solidago altissima). BMC Plant Biol 19: 1-12. 


\section{Table and Figure legends}

544 Figure 1 Measured and simulated growth dynamics of $C$. elatum in A) isolation and B) competition

with $P$. sapidus. Points describe empirical measurements, and lines are the corresponding

simulation model output. Light shades represent the control treatment, while darker shades

represent the respective stress treatments (stressed, T, or primed and stressed, PT). Error bars show

the standard error of the mean of the observed data. Examples at the right show the corresponding output of the cellular automaton model at day eight.

Figure 2 Priming benefit of $C$. elatum. A) Simulation of the priming benefit of $C$. elatum over time in

isolation or competition. B) Comparison of the priming benefit of $C$. elatum in isolation or

competition with one of five other soil fungi. Values represent the observed benefit at the last day

of measurements and the simulated benefit for the same day.

Figure 3 Priming benefit of $C$. elatum in competition with an artificial species. Benefits are shown for

different trait combinations eight days after stress treatment. Levels of susceptibility correspond to

different lengths of a stress-induced lag phase: low $=0.5$ days, intermediate $=1.5$ days, high $=2$

days, and levels of primeability correspond to the reduction of this lag phase under priming

conditions: low $=25 \%$, intermediate $=50 \%$, high $=100 \%$.

559 Figure 4 Competitive shift of $C$. elatum in competition with an artificial species. The shifts in

560 competition are shown eight days after the stress treatment. Red shades indicate a shift in favor of

561 C. elatum, and blue shades a shift favoring its competitor. Photos show exemplary pairwise cultures

562 grown in the laboratory: Each pair is assigned the respective shift of competition predicted by the

563 simulation model according to the parameter values of the competitor and growth of $C$. elatum. The

564 pairs shown are C. elatum (red) competing against (blue) 1. F. redolens, 2. T. angustata, 3. P. sapidus

565 4. F. oxysporum, 5. M. elongata. M. elongata is fast growing and not primeable, and is not 
566 represented in the visualized parameter space. Levels of susceptibility correspond to different

567 lengths of a stress-induced lag phase: low $=0.5$ days, intermediate $=1.5$ days, high $=2$ days, and

568 levels of primeability correspond to the reduction of this lag phase under priming conditions: low =

$56925 \%$, intermediate $=50 \%$, high $=100 \%$.

570 Table 1 Experimentally measured values of growth rates and their relative changes and lag phases

571 after a stress stimulus. The significance levels between growth rates were assessed by a paired t-

572 test $* P \leq 0.05, * * P \leq 0.01$, relative changes of 1 indicated a non-significant change in growth rate.

573 Abbreviations: C: control treatment without stress, P: primed treatment with a mild stress, T:

574 triggered treatment with a strong stress, PT: primed and triggered treatment.

575 Table 2 Model parameters and their description. 
A
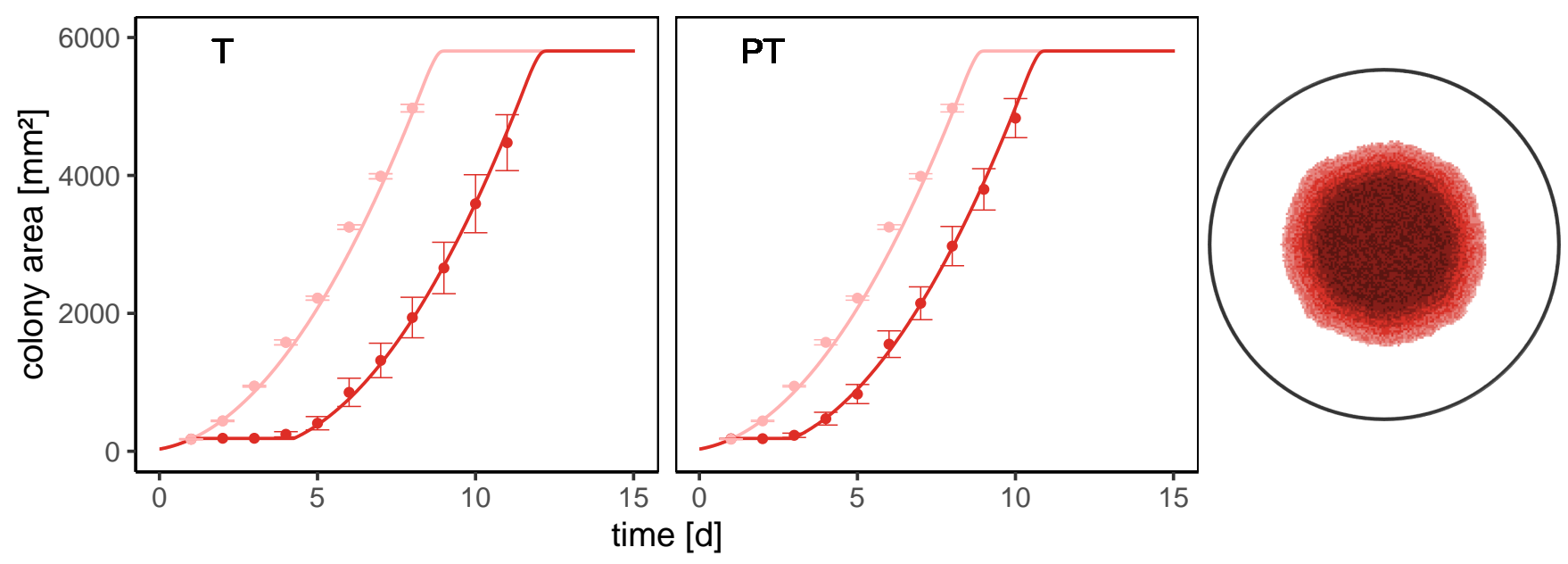

B
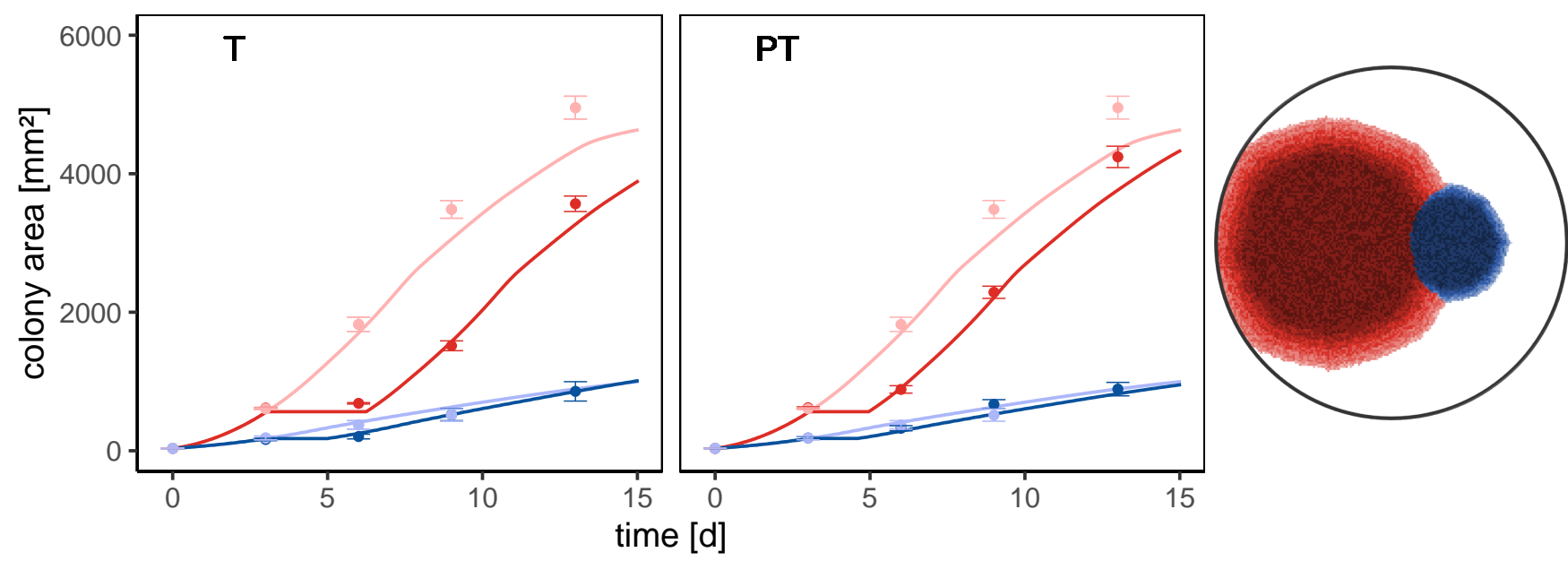


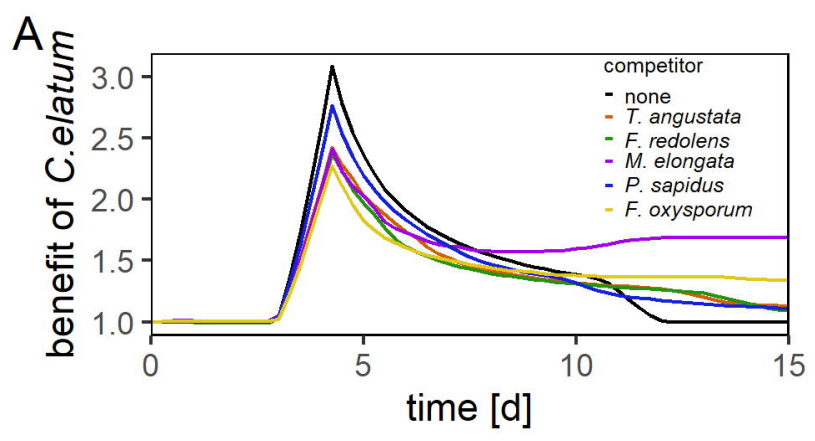

B

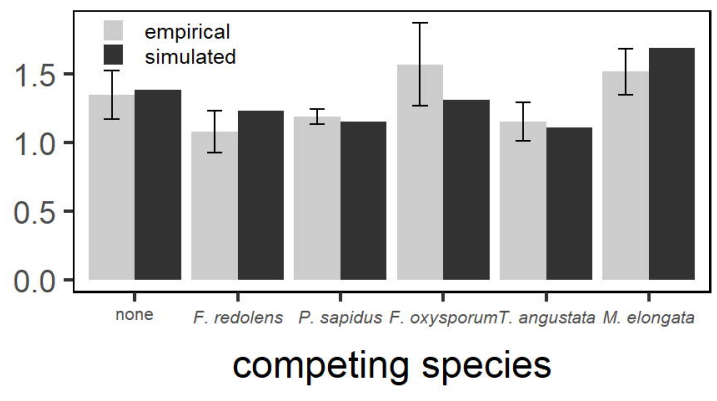




\section{primeability of competitor}

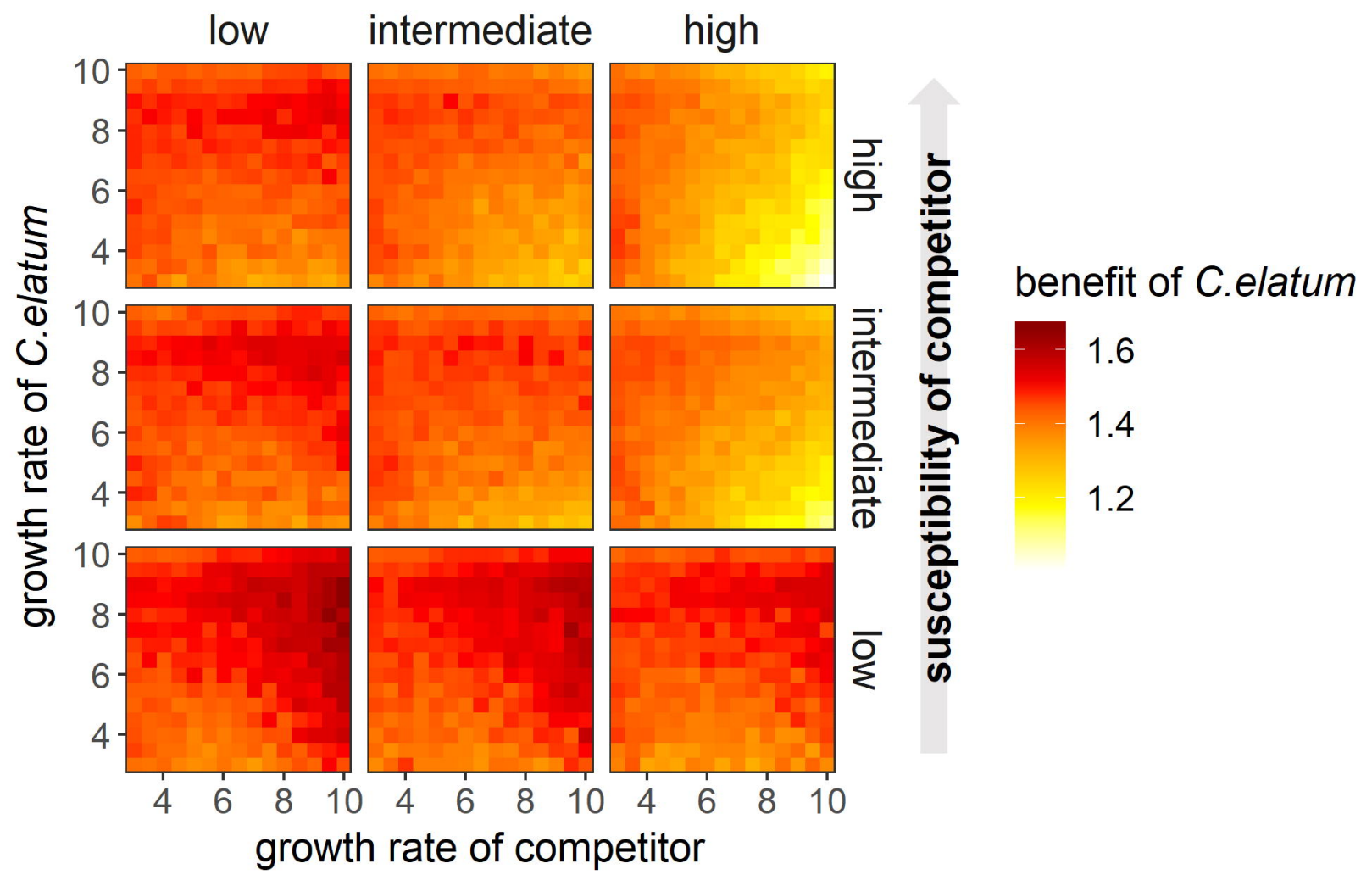




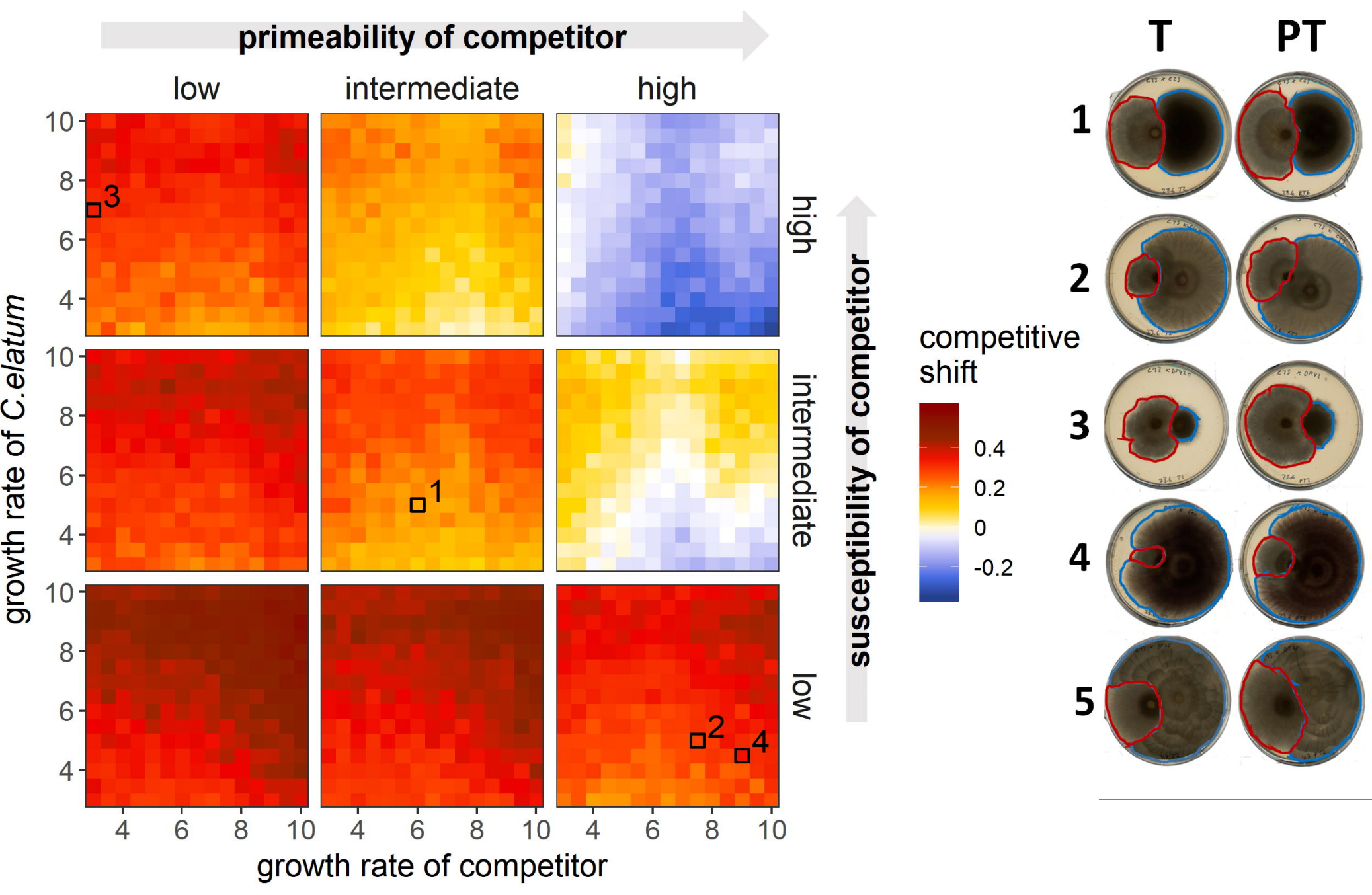

